\title{
Assessment of Serum (YKL-40) As an Early Diagnostic Marker of Diabetic Nephropathy in Patients with Type 2 Diabetes Mellitus \\ Mohamad Ahmed Shaaban ${ }^{1}$, Mohamad Abd El-raoof Korany ${ }^{1}$, Rania Mohamad Azmy El-Shazly², Mohammad Ali Nasr Ibrahem ${ }^{* 3}$ \\ Departments of ${ }^{1}$ Internal Medicine and ${ }^{2}$ Medical Biochemistry, \\ Faculty of Medicine, Menoufia University, Egypt, ${ }^{3} \mathrm{Kfr}$ Elshiekh Liver Centre, Kfr Elshiekh-Egypt.
}

*Correspondence author: Mohammad Ali Nasr Ibrahem, Mobile: (+20)01009414389, E-mail: drmohamed183@gmail.com

\begin{abstract}
Background: YKL-40 is an inflammatory marker, a positive association between elevated circulating YKL-40 levels and increasing levels of albuminuria, which have been described in patients with type 1 and type 2 diabetes indicating a role of YKL-40 in the progressing vascular damage resulting in microvascular disease.

Objectives: To assess serum YKL-40 as an early diagnostic marker of diabetic nephropathy in type 2 diabetes mellitus. Patients and Methods: 70 patients with type 2 diabetes mellitus (46 males and 24 females) were included in this study, another 19 healthy volunteers (12 males and 7 females) were chosen as a control group (group I). Patients were further classified according to their Albumin/creatinine ratio into three groups: group II; Diabetic normoalbuminuric, group III; Diabetic microalbuminuric and group IV; Diabetic macroalbuminuric. All subjects (patients and controls) participating in the study were subjected to full history and clinical examination and routine laboratory investigations and also specific laboratory investigations include glycated hemoglobin (HbA1c), urine creatinine, microalbumin, albumin creatinine ratio, and serum YKL 40.

Results: The present study showed that serum YKL-40 significantly increased in diabetic patients than in the control group. In addition, there was a significant increase of YKL-40 in the macroalbuminuric group compared to the normoalbuminuric group and microalbuminuric group and a significant increase of YKL-40 in a microalbuminuric group compared to the normoalbuminuric group.
\end{abstract}

Conclusion: The serum levels of YKL-40 in type 2 DM were significantly higher than in normal patients, and its level increased with increasing urinary albumin excretion rate.

Keywords: Albumin/Creatinine Ratio, Albuminuria, Diabetes mellitus, Diabetic nephropathy, YKL-40.

\section{INTRODUCTION}

Diabetes mellitus is a metabolic disease characterized by defective insulin secretion, insulin action, or both ${ }^{(1)}$. Diabetic nephropathy is the most common cause of end-stage renal disease. Microalbuminuria is a widely used indicator for diabetic nephropathy; its diagnostic accuracy is limited because structural damage might precede albumin excretion. Therefore, sensitive and specific biomarkers for early detection of diabetic nephropathy are needed (2). YKL-40 serum levels are elevated in both patients with type 1 and type 2 diabetes ${ }^{(\mathbf{3})}$.

The aim of this work was to assess serum YKL-40 as an early diagnostic marker of diabetic nephropathy in type 2 diabetes mellitus.

\section{PATIENTS AND METHODS}

70 patients with type 2 diabetes mellitus (46 males and 24 females) were included in this study, and 19 healthy volunteers (12 males and 7 females) were chosen as control (Group I) without a history of arterial hypertension, diabetes, neoplastic, cardiovascular, inflammatory, renal, lung, endocrine or central nervous system disorders. None of these subjects was under medical treatment. Patients were further classified according to their Albumin/creatinine ratio into three groups: Group II: Diabetic normoalbuminuric. It included 26 patients with type 2 diabetes with normal albuminuria (15 males and 11 females), the albumin/creatinine ratio in these patients was less than $30 \mathrm{mg}$ albumin/g creatinine, Group III: Diabetic microalbuminuric. It included 22 patients with type 2 diabetes with microalbuminuria (15 males and 7 females), the albumin/creatinine ratio in these patients was 30 - $300 \mathrm{mg}$ albumin/g creatinine, and Group IV: Diabetic macroalbuminuric. It included 22 patients with type 2 diabetes with macroalbuminuria (16 males and 6 females). The albumin/creatinine ratio in this group was more than $300 \mathrm{mg}$ albumin/g creatinine. Patients were recruited from the Internal Medicine Department and Endocrinology Outpatient Clinic of Menoufya University Hospital through the period from March to December 2019.

Ethical and patients' approval: Before starting the study, signed informed written consents were obtained from all subjects enrolled in the study as well as approval of the Ethics Committee of Menoufya University Hospitals was obtained.

Inclusion criteria: Patients were included in the study if their fasting plasma glucose (FPG) was $\geq 126 \mathrm{mg} / \mathrm{dl}$, $\mathrm{PPPG} \geq 200 \mathrm{mg} / \mathrm{dl}$ or $\mathrm{HbA} 1 \mathrm{c} \geq 6.5 \%$ according to American Diabetic Association (ADA) guidelines ${ }^{(4)}$. 
Exclusion criteria: Patients with active urinary tract infection, patients with abnormal urinary sediment, patients with a history of other renal diseases except for diabetic nephropathy, patients with active or chronic infection, patients with cancer, patients with any active or chronic inflammatory conditions, patients with severe liver dysfunction, patients with uncontrolled thyroid disorders and pregnant and lactating females.

All participants (patients and controls) in the study were subjected to the following: Thorough history taking, physical examination, laboratory investigations including complete blood count (CBC), fasting blood glucose (FBG), postprandial blood glucose (PPBG), glycated hemoglobin (HbA1c), serum aspartate transaminase (AST), serum alanine transaminase (ALT), serum albumin, serum total cholesterol, serum triglycerides, high-density lipoprotein (HDL), low-density lipoprotein (LDL), very low-density lipoprotein (VLDL), serum creatinine, urine creatinine, microalbumin in urine, albumin $\mathrm{mg} / \mathrm{g}$ creatinine (Albumin creatinine ratio) and Serum YKL - 40. Blood samples were obtained from all patients on their routine clinical follow-up visits. After 12 hours of fasting, venous blood was withdrawn from patients as follows, $10 \mathrm{~mL}$ in a dry clean tube were left to clot for 20 minutes at $37^{\circ} \mathrm{C}$ then centrifuged at $3000 \mathrm{xg}$ for 10 minutes. Dividing the serum into separate tubes. $2 \mathrm{ml}$ in a dry clean tube was stored at $20^{\circ} \mathrm{C}$ until assay. It is used to estimate YKL40. The rest of the serum was used for estimation of ALT, AST, Albumin, creatinine, FBS, cholesterol, triglycerides, HDL.

After 2 hours, postprandial venous blood was withdrawn from patients as follows, $3 \mathrm{ml}$ in a dry clean tube were left for 20 minutes at $37^{\circ} \mathrm{C}$ then centrifuged at $3000 \mathrm{xg}$ for 10 minutes. The serum was used for estimation of postprandial blood sugar. $2 \mathrm{ml}$ in EDETA tube were used for doing $\mathrm{CBC}$ and for estimation of HbA1c. Urine samples: Ten milliliters $(10 \mathrm{~mL})$ of morning urine samples were collected in a clean container for immediate estimation of creatinine and microalbumin.

FPG, PPPG, AST, ALT, HbA1c, serum albumin, serum total cholesterol, serum triglycerides, highdensity lipoprotein (HDL), low-density lipoprotein (LDL), very low-density lipoprotein (VLDL), uea, and creatinine, were measured by Au680 autoanalyzer provided by Beckman Coulter/Olympus, Japan ${ }^{(5)}$. Serum YKL-40 was determined by The Human YKL40 ELISA (Enzyme-Linked Immunosorbent Assay) procedure using BIORAD ELISA reader Diagnostics (GERMANY) using SUNRED ELISA KIT China ${ }^{(6,7)}$.

Routine pelvic abdominal ultrasound was done for all participants to exclude other renal diseases and hepatic patients.

\section{Statistical analysis}

Data collected throughout history, basic clinical examination, laboratory investigations, and outcome measures were coded, entered, and analyzed using Microsoft Excel software. Data were then imported into Statistical Package for the Social Sciences (SPSS version 20.0) (IBM company, 233 South Wacker Drive, $11^{\text {th }}$ Floor Chicago, Illinois 60606-6307, United States of America (U.S.A)) software for analysis. Two types of statistics were done: Descriptive, e.g. range, minimum, maximum, mean, and standard deviation ( \pm SD) for quantitative data.

Analytical, Student's t-test was used to collectively indicate the presence of any significant difference between two groups for a normally distributed quantitative variable. One away ANOVA ( $F$ test): A one-way analysis of variance (ANOVA) is a single test used to indicate the presence of any significant difference between several groups for a normally distributed quantitative variable. Pearson's correlation coefficient (r) test was used for correlating data. Negative correlations were expressed in (-) symbol. Significance of results, the corresponding Pvalue for each test was directly computed by the microprocessor. A significant difference was considered when $\mathrm{P}$ is $\leq 0.05^{(\boldsymbol{8})}$.

\section{RESULTS}

The present work included 70 patients with type 2 diabetes mellitus (46 males, and 24 females). In addition, 19 healthy volunteers were chosen as control (12 males and 7 females). Table (1) showed descriptive data of study groups.

As expected, the patient group showed higher FPG, PPPG, and HbA1c than the control group. As regards serum creatinine, there was a highly significant difference between control, and microalbuminuric group, control, and macroalbuminuric group, normoalbuminuric and macroalbuminuric group, normoalbuminuric and microalbuminuric group, and microalbuminuric and macroalbuminuric group. However, there was no significant difference between control and normoalbuminuric group. Regarding albumin-creatinine ratio $(\mathrm{mg} / \mathrm{g})$ there was no significant difference between control and normoalbuminuric group, control, and microalbuminuric group, and normoalbuminuric and microalbuminuric group and there is a highly significant difference between control and macroalbuminuric group, normoalbuminuric and macroalbuminuric group, and microalbuminuric and macroalbuminuric group. Figure (1) showed the mean of albumin creatinine ratio in control, normoalbuminuric, microalbuminuric, and macroalbuminuric groups.

In the present study, there was lower eGFR (mg/L) in type 2 diabetic patients than healthy controls. In addition, patients with macroalbuminuria had lower eGFR than microalbuminuric and normoalbuminuric. The microalbuminuric patients had a significantly lower eGFR than those with normoalbuminuria. 
Table (1): Descriptive data of study groups

\begin{tabular}{|c|c|c|c|c|c|}
\hline & Control & Normoalbuminuria & Microalbuminuria & Macroalbuminuria & $P$ value \\
\hline $\mathrm{N}^{* *}$, total & 19 & 26 & 22 & 22 & \\
\hline & Mean \pm SD & Mean \pm SD & Mean \pm SD & Mean \pm SD & \\
\hline Age (years) & $47.84 \pm 5.10$ & $52.88 \pm 4.93$ & $54.54 \pm 3.01$ & $55.77 \pm 3.57$ & $0.001 *$ \\
\hline WBCs** $\left(\mathrm{X10}^{3} / \mathrm{mm}^{3}\right)$ & $5.80 \pm 1.94$ & $6.24 \pm 1.49$ & $7.83 \pm 1.83$ & $7.65 \pm 2.06$ & $0.003^{*}$ \\
\hline $\begin{array}{l}\mathrm{RBCs}^{* *}\left(\mathrm{X100}^{3} \text { cells } /\right. \\
\mu \mathrm{L})\end{array}$ & $4.6 \pm 0.46$ & $4.32 \pm 0.57$ & $4.36 \pm 0.48$ & $3.85 \pm 0.466$ & $0.001^{*}$ \\
\hline Hemoglobin (g/dL) & $13.02 \pm 0.88$ & $13.15 \pm 1.48$ & $12.30 \pm 1.08$ & $11.65 \pm 1.16$ & $0.001 *$ \\
\hline Platelets $\left(\mathrm{X10}^{3} / \mathrm{mm}^{3}\right)$ & $259.11 \pm 55.14$ & $276.77 \pm 16.70$ & $231.64 \pm 7.98$ & $244.86 \pm 7.80$ & 0.250 \\
\hline $\mathbf{A L T}^{* *}(\mathbf{U} / \mathbf{L})$ & $23.36 \pm 5.62$ & $23.88 \pm 4.38$ & $42.54 \pm 8.15$ & $40.22 \pm 2.62$ & $0.001^{*}$ \\
\hline $\mathbf{A S T} * *(\mathbf{U} / \mathbf{L})$ & $24.05 \pm 5.86$ & $30.57 \pm 5.09$ & $40.81 \pm 5.37$ & $39.68 \pm 8.27$ & $0.001 *$ \\
\hline FBG** (mg/dL) & $96.31 \pm 5.18$ & $180.38 \pm 9.17$ & $199.91 \pm 6.63$ & $229.45 \pm 6.06$ & $0.001 *$ \\
\hline PPBG** (mg/dL) & $110.00 \pm 6.76$ & $269.31 \pm 8.16$ & $326.09 \pm 8.24$ & $351.86 \pm 6.07$ & $0.001^{*}$ \\
\hline HBA1c** $(\%)$ & $5.03 \pm 0.29$ & $8.40 \pm 1.76$ & $10.22 \pm 1.72$ & $10.51 \pm 1.93$ & $0.001 *$ \\
\hline Cholesterol (mg/dL) & $154.84 \pm 22.3$ & $221.50 \pm 55.45$ & $282.82 \pm 49.13$ & $281.64 \pm 51.31$ & $0.001 *$ \\
\hline Triglycerides (mg/dL) & $114.37 \pm 17.16$ & $203.50 \pm 52.93$ & $248.86 \pm 46.63$ & $251.05 \pm 66.50$ & $0.001 *$ \\
\hline HDL** (mg/dL) & $44.57 \pm 7.99$ & $45.50 \pm 2.172$ & $40.90 \pm 8.38$ & $40.90 \pm 10.98$ & 0.28 \\
\hline VLDL** (mg/dL) & $22.89 \pm 3.46$ & $40.70 \pm 10.58$ & $49.77 \pm 9.32$ & $50.20 \pm 13.30$ & $0.001^{*}$ \\
\hline $\mathrm{LDL}^{* *}(\mathrm{mg} / \mathrm{dL})$ & $87.37 \pm 18.15$ & $135.30 \pm 9.00$ & $192.14 \pm 5.89$ & $190.52 \pm 5.97$ & $0.001^{*}$ \\
\hline $\begin{array}{l}\text { Serum } \\
\text { creatinine(mg/dL) }\end{array}$ & $0.91 \pm 0.21$ & $0.97 \pm 0.20$ & $1.15 \pm 0.23$ & $1.62 \pm 0.175$ & $0.001 *$ \\
\hline Serum Albumin(g/d) & $4.33 \pm 0.50$ & $4.04 \pm 0.36$ & $3.84 \pm 0.32$ & $3.30 \pm 0.33$ & $0.001 *$ \\
\hline eGFR $* *(\mathrm{mg} / \mathrm{L})$ & $93.05 \pm 13.98$ & $79.73 \pm 13.19$ & $67.36 \pm 12.11$ & $45.09 \pm 5.02$ & $0.001^{*}$ \\
\hline $\mathbf{A C R}^{* *}(\mathbf{m g} / \mathrm{g})$ & $17.50 \pm 4.66$ & $21.67 \pm 5.15$ & $138.29 \pm 5.75$ & $2069.3 \pm 449.11$ & $0.001^{*}$ \\
\hline YKL-40 (ng/mL) & $24.40 \pm 8.17$ & $71.17 \pm 7.15$ & $193.12 \pm 8.91$ & $578.6 \pm 74.51$ & $0.001^{*}$ \\
\hline
\end{tabular}

* Significant $* * \mathrm{~N}$ :number, WBCs: white blood cells, RBCs: red blood corpuscle, ALT: alanine transaminase, AST: aspartate transaminase, FBG: fasting blood glucose, PPBG: post prandial blood glucose, HbA1c: glycated hemoglobin, HDL: highdensity lipoprotein, VLDL: very low-density lipoprotein, LDL: low-density lipoprotein, ACR: albumin creatinine ratio.

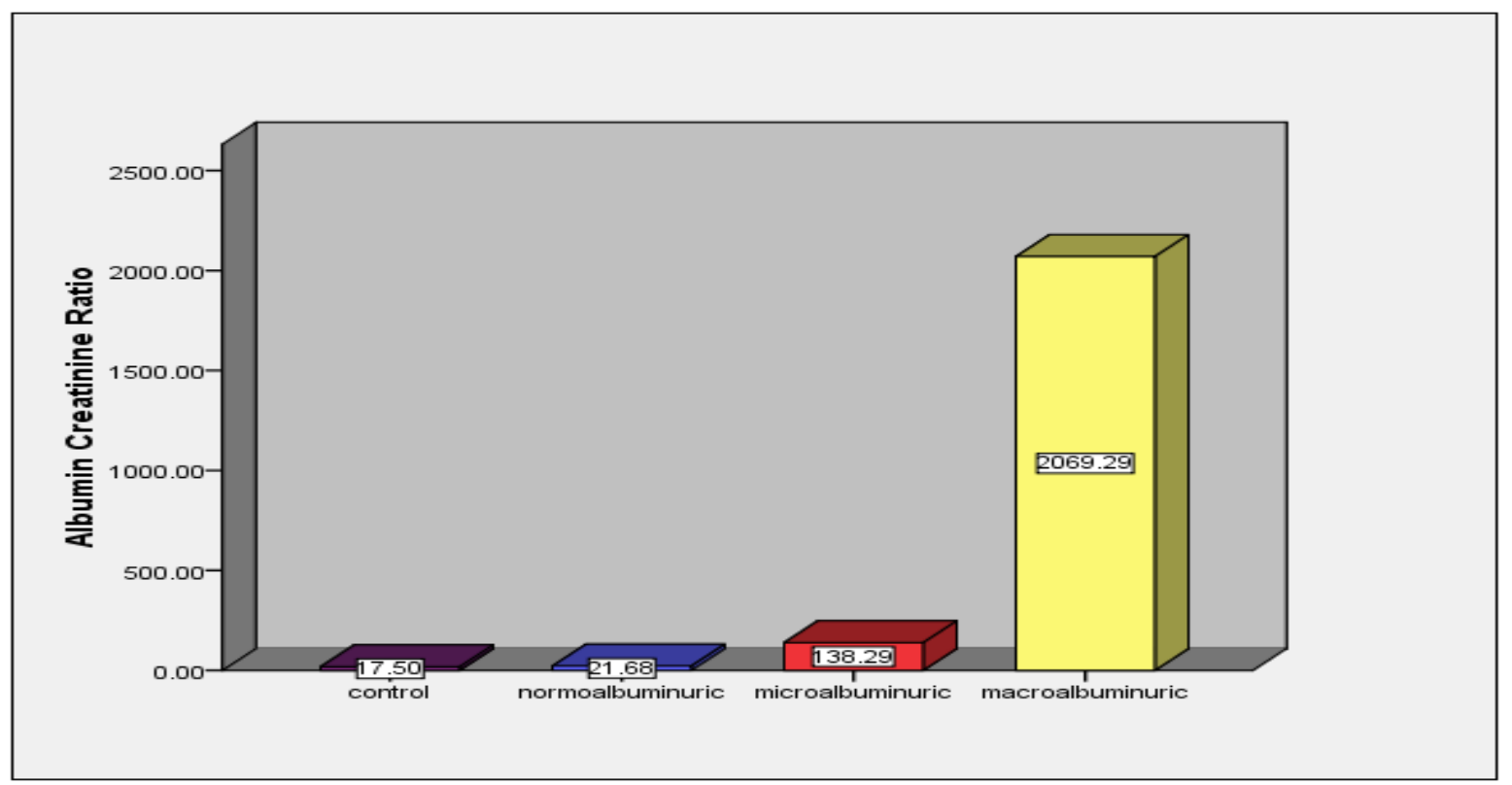

Figure (1): The figure show the mean of albumin creatinine ratio in control, normoalbuminuric, microalbuminuric and macroalbuminuric groups.

The present study showed that serum YKL-40 significantly increased in diabetic patients than in the control group. In addition, there was a significant increase of YKL-40 in the macroalbuminuric group compared to the normoalbuminuric group and microalbuminuric group and a significant increase of YKL-40 in the microalbuminuric group compared to the normoalbuminuric group (Figure 2). 


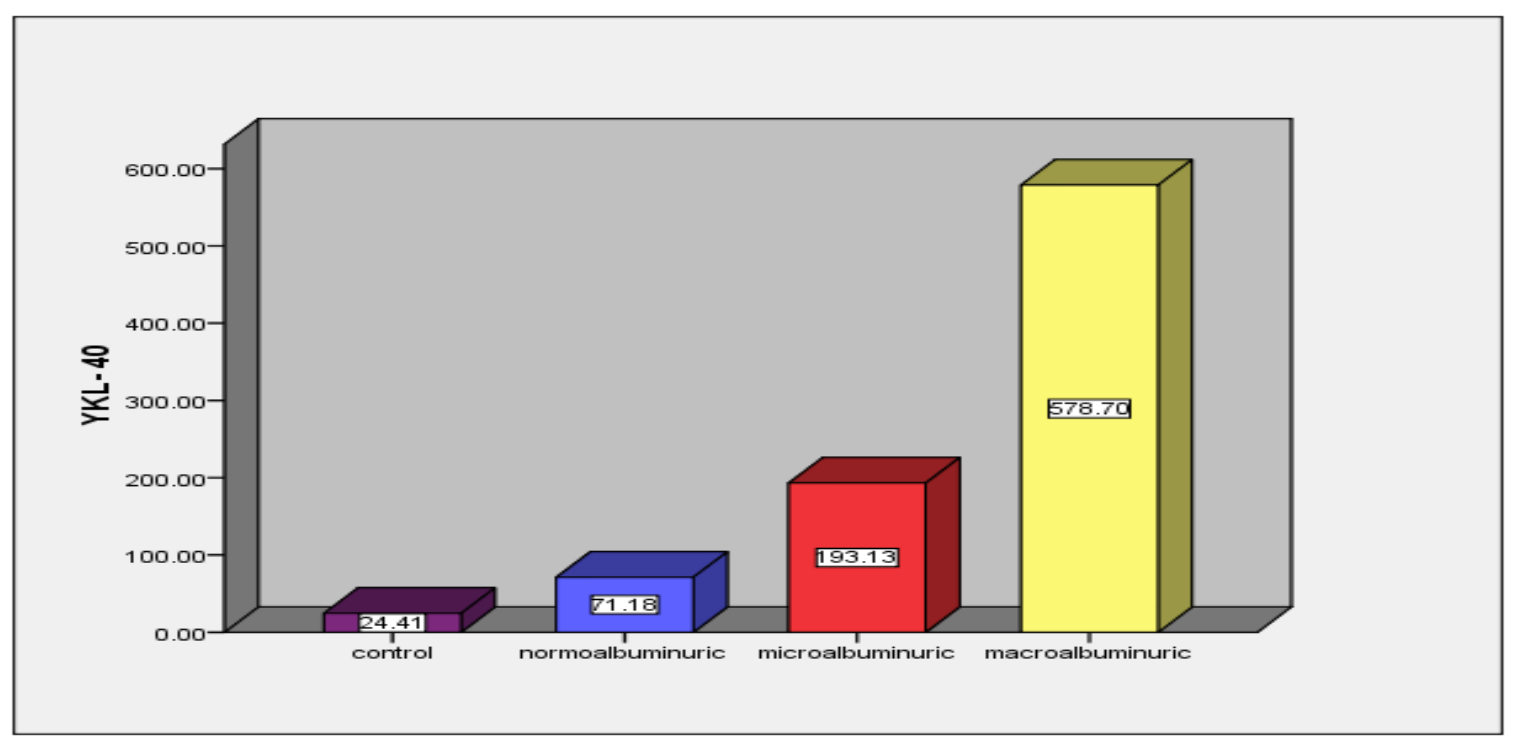

Figure (2): The figure show the mean of YKL $40(\mathrm{ng} / \mathrm{mL})$ in control, normoalbuminuric, microalbuminuric and macroalbuminuric groups.

The present study, showed that there was a positive and significant correlation between YKL-40 and eGFR ( $\mathrm{r}$ $=0.547, \mathrm{P}=0.008)$, and negative and significant correlation between YKL-40 and serum creatinine $(\mathrm{r}=-0.531, \mathrm{P}$ $=0.01)$ in microalbuminuric group (Table 2$)$.

Table (2): Correlations of YKL40 with other parameters in the microalbuminuric group.

\begin{tabular}{|c|c|c|}
\hline & $\mathbf{r}^{* *}$ & P-value \\
\hline Age (Years) & 0.120 & 0.59 \\
\hline WBCs* $\left(\mathrm{X10}^{3} / \mathrm{mm}^{3}\right)$ & 0.129 & 0.56 \\
\hline $\mathrm{RBCs}^{*}\left(\mathrm{X} \mathrm{100}{ }^{3}\right.$ cells $\left./ \mu \mathrm{L}\right)$ & 0.145 & 0.51 \\
\hline Hemoglobin (g/dL) & 0.291 & 0.19 \\
\hline Platelets $\left(\mathrm{X10}^{3} / \mathrm{mm}^{3}\right)$ & -0.121 & 0.59 \\
\hline ALT* $(\mathbf{U} / \mathbf{L})$ & 0.139 & 0.53 \\
\hline $\mathrm{AST}^{*}(\mathrm{U} / \mathrm{L})$ & 0.015 & 0.94 \\
\hline FBG* (mg/dL) & -0.019 & 0.93 \\
\hline PPBG* (mg/dL) & 0.183 & 0.41 \\
\hline HBA1c* $(\%)$ & 0.173 & 0.44 \\
\hline Cholesterol (mg/dL) & -0.187 & 0.40 \\
\hline Triglycerides (mg/dL) & -0.084 & 0.71 \\
\hline HDL* $^{*}(\mathrm{mg} / \mathrm{dL})$ & 0.079 & 0.72 \\
\hline VLDL* (mg/dL) & -0.084 & 0.71 \\
\hline $\mathrm{LDL}^{*}(\mathrm{mg} / \mathrm{dL})$ & -0.178 & 0.42 \\
\hline Serum creatinine (mg/dL) & -0.531 & 0.01 \\
\hline Serum Albumin (g/dL) & 0.035 & 0.87 \\
\hline eGFR* (mg/L) & 0.547 & 0.008 \\
\hline Albumin creatinine ratio & -0.246 & 0.269 \\
\hline
\end{tabular}

** r: correlation coefficient,* WBCs: white blood cells, RBCs: red blood corpuscle, ALT: alanine transaminase, AST: aspartate transaminase, FBG: fasting blood glucose, PPBG: postprandial blood glucose, HbA1C: Glycated hemoglobin, HDL: high-density lipoprotein, VLDL: very low-density lipoprotein, LDL: low-density lipoprotein, eGFR: estimated glomerular filtration rate.

In the present study, there was a positive and significant correlation between YKL-40 and ALT ( $\mathrm{r}=0.453$, $\mathrm{P}=0.03$ ) in the macroalbuminuric group (Table 3). 
Table (3): Correlations of YKL40 with other parameters in the macroalbuminuric group

\begin{tabular}{|c|c|c|}
\hline & $\mathbf{r}^{* *}$ & P-value \\
\hline Age (Years) & -0.144 & 0.52 \\
\hline WBCs** $\left(\mathrm{X} 10^{3} / \mathrm{mm}^{3}\right)$ & 0.145 & 0.52 \\
\hline $\mathbf{R B C s}^{* *}\left(\mathrm{X} \mathrm{100^{3 }}\right.$ cells $\left./ \mu \mathrm{L}\right)$ & -0.127 & 0.57 \\
\hline Hemoglobin (g/dL) & -0.059 & 0.79 \\
\hline Platelets $\left(\mathrm{X} 10^{3} / \mathrm{mm}^{3}\right)$ & 0.029 & 0.89 \\
\hline $\mathbf{A L T} * *(\mathbf{U} / \mathbf{L})$ & 0.453 & $0.03 *$ \\
\hline $\mathrm{AST}^{* *}(\mathbf{U} / \mathbf{L})$ & 0.380 & 0.08 \\
\hline FBG** (mg/dL) & 0.227 & 0.31 \\
\hline PPBG** (mg/dL) & 0.106 & 0.63 \\
\hline HBA1c** $(\%)$ & 0.251 & 0.26 \\
\hline Cholesterol (mg/dL) & 0.201 & 0.37 \\
\hline Triglycerides (mg/dL) & -0.038 & 0.86 \\
\hline HDL** (mg/dL) & -0.071 & 0.75 \\
\hline VLDL** $(\mathrm{mg} / \mathrm{dL})$ & -0.038 & 0.86 \\
\hline $\mathrm{LDL}^{* *}(\mathrm{mg} / \mathrm{dL})$ & 0.223 & 0.31 \\
\hline Serum creatinine (mg/dL) & -0.085 & 0.70 \\
\hline Serum Albumin (g/dL) & 0.079 & 0.72 \\
\hline eGFR** $(\mathrm{mg} / \mathrm{L})$ & -0.091 & 0.68 \\
\hline Albumin creatinine ratio & 0.094 & 0.67 \\
\hline
\end{tabular}

* Significant ** r: correlation coefficient, WBCs: white blood cells, RBCs: red blood corpuscle, ALT: alanine transaminase, AST: aspartate transaminase, FBG: fasting blood glucose, PPBG: postprandial blood glucose, HbA1c: Glycated hemoglobin, HDL: high-density lipoprotein, VLDL: very low-density lipoprotein, LDL: low-density lipoprotein, eGFR:estimated glomerular filtration rate.

In the present study, ROC analysis of data from 70 samples: Cut off, sensitivity and specificity of YKL-40, as a marker for diagnosis of diabetic nephropathy was calculated using albumin creatinine ratio (ACR) as a reference, for YKL-40. The area under the ROC (AUROC) was estimated to be 0.912, and the optimal cut-off value for YKL-40 was found to be $57.1 \mathrm{ng} / \mathrm{ml}$, had a diagnostic sensitivity of $94.2 \%$, and specificity of $90.7 \%$, which mean a very good diagnostic accuracy of YKL-40 as a marker for diagnosis of diabetic nephropathy. On the other hand, we found that ACR (mg/g) at a cut off value $30 \mathrm{mg} / \mathrm{g}$ had a diagnostic sensitivity of $73.5 \%$ and specificity of $50 \%$ the area under the ROC (AUROC) was estimated to be 0.67 (Figure 3).

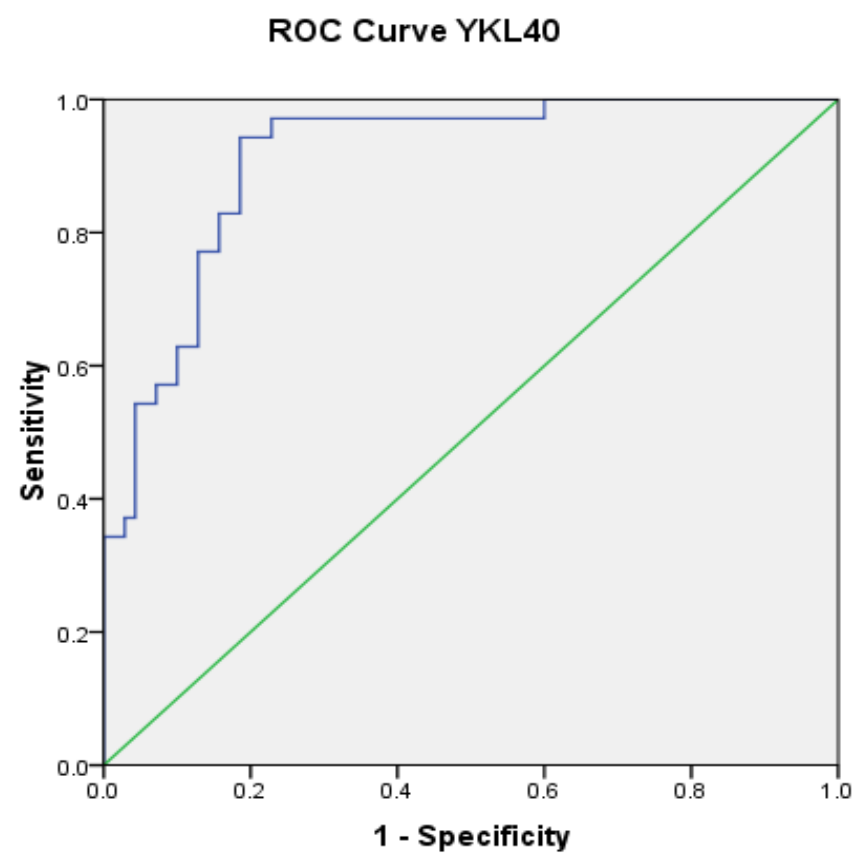

Figure (3): Receiver operating characteristics (ROC) curve of serum YKL 40 in diagnosis of diabetic nephropathy. 


\section{DISCUSSION}

Diabetic nephropathy one of the microvascular complications of diabetes is a leading cause of diabetes-related morbidity and mortality worldwide and its progression could be retarded by preventing some of the vital risk factors such as hyperglycemia, dyslipidemia, hypertension, and by blocking the renin-angiotensin-aldosterone system ${ }^{\left({ }^{(9)}\right.}$.

Albuminuria or Albumin / Creatinine Ratio (ACR) has been considered for the last three decades as the gold standard diagnostic and prognostic biomarker for diabetic nephropathy onset and progression $^{(2)}$.

Association of mild albuminuria with progressive renal disease has been highly challenged, except in patients of progression of albuminuria. Albuminuria also lacks specificity and sensitivity as a prognostic biomarker for progressive diabetic nephropathy ${ }^{(\mathbf{1 0})}$, as diabetic nephropathy is sometimes able to progress without an increase in albuminuria and even in the presence of normoalbuminuria ${ }^{(11)}$. In the present study, the mean fasting blood glucose $(\mathrm{mg} / \mathrm{dl})$ in control, normoalbuminuric, microalbuminuric, and macroalbuminuric was 96.31 $\pm 5.18,180.38 \pm 39.17,199.91 \pm 63.63$, and $229.45 \pm$ 60.06 respectively. There was a highly significant difference between control and normoalbuminuric groups, control, and microalbuminuric groups, control and macroalbuminuric groups and normoalbuminuric and macroalbuminuric groups and significant difference between microalbuminuric and macroalbuminuric groups and no significant difference between normoalbuminuric and microalbuminuric groups. This result is in agreement with Elsayed et al. ${ }^{(12)}$ who found that the mean FBS $(\mathrm{mg} / \mathrm{dl})$ was $85.4 \pm 8.88,130.1 \pm 20.36,221.9 \pm 58.49$ and $229.4 \pm 56.36$ in control, normoalbuminuric, microalbuminuric, and macroalbuminuric groups respectively. There was a significant difference between the control group and normoalbuminuric group and there was a highly significant difference between control and microalbuminuric group and between control and macroalbuminuric group and between normoalbuminuric and microalbuminuric group and between normoalbuminuric and macroalbuminuric group.

In the present study, the mean postprandial blood glucose $(\mathrm{mg} / \mathrm{dl})$ in control, normoalbuminuric, microalbuminuric, and macroalbuminuric was 110.00 $\pm 6.76,269.31 \pm 83.16,326.09 \pm 78.24$, and $351.86 \pm$ 61.07 respectively. There was a highly significant difference between control and normoalbuminuric group, control and microalbuminuric group, control and macroalbuminuric group, normoalbuminuric and microalbuminuric group and normoalbuminuric and macroalbuminuric group. While, there was no significant difference between microalbuminuric and macroalbuminuric groups. This result is in agreement with Umapathy et al. ${ }^{(13)}$ who found that the mean postprandial blood glucose (PPBG) $(\mathrm{mg} / \mathrm{dl})$ was 120.2 $\pm 13.7,211.7 \pm 47.4$, and $240.6 \pm 47.2,243.6 \pm 61.45$ in control, normoalbuminuric, microalbuminuric, and macroalbuminuric groups respectively. There was a highly significant difference between control and all other groups and there was a highly significant difference between normoalbuminuric group and microalbuminuric and macroalbuminuric groups. However, there was no significant difference between microalbuminuric and macroalbuminuric group.

In the present study, the mean albumin creatinine ratio $(\mathrm{mg} / \mathrm{g})$ in control, normoalbuminuric, microalbuminuric, and macroalbuminuric groups was $17.50 \pm 4.66,21.67 \pm 5.15,138.29 \pm 45.75$, and $2069.3 \pm 1449.11$ respectively. There was no significant difference between control and normoalbuminuric groups, control and microalbuminuric groups and normoalbuminuric and microalbuminuric groups and there was a highly significant difference between, control and macroalbuminuric groups, normoalbuminuric and macroalbuminuric groups, and microalbuminuric and macroalbuminuric groups. Sueud et al. ${ }^{(14)}$ found that the mean albumin creatinine ratio $(\mathrm{mg} / \mathrm{g})$ was $15.2 \pm 8.3,19.7 \pm 7.4,74.4 \pm 41.3$ and $1077.2 \pm 171.1$ in control, normoalbuminuric, microalbuminuric, and macroalbuminuric groups respectively. There was no significant difference between the control group and the other study groups and no significant difference between normoalbuminuric group and the other study groups. However, there was a significant difference between microalbuminuric group and the other study groups and between the macroalbuminuric group and the other study groups.

In the present study, the mean YKL-40 in control, normoalbuminuric, microalbuminuric, and macroalbuminuric was $24.40 \pm 18.17,71.17 \pm 37.15$, $193.12 \pm 84.91$, and $578.6 \pm 174.51$ respectively. There was a highly significant difference between control and microalbuminuric groups, control and macroalbuminuric groups, normoalbuminuric and macroalbuminuric groups, normoalbuminuric and microalbuminuric groups and microalbuminuric and macroalbuminuric groups. However, there was no significant difference between control and normoalbuminuric groups. This result is in agreement with Umapathy et al. (13) who found that the circulatory levels of YKL-40 were 2.7 (0.74-4.63),4.0 (2.62-5.52), $7.381(4.09-12.79)$ and $14.35(5.1-36.36)$ in control, normoalbuminuric, microalbuminuric, and macroalbuminuric groups respectively. There was a significant difference between control and all other groups. However, there was a highly significant difference between normoalbuminuric group and microalbuminuric and macroalbuminuric groups, and 
significant difference between microalbuminuric and macroalbuminuric groups. Han et al. ${ }^{(15)}$ found that the median YKL-40 (ng/ml) was 27.6 (24.5-29.3), 44.6 (41.1-48.8), 67.5 (61.9-70.7) and 72.5 (60.576.3) in control, normoalbuminuric, microalbuminuric, and macroalbuminuric groups respectively. There was a highly significant difference between the studied groups.

In the present study, there was a positive and significant correlation between YKL-40 and triglycerides, and VLDL in the control group. In addition, there was a positive and non-significant correlation between YKL-40 and $\mathrm{HbAlc}$ in the macroalbuminuric group. These results are in disagreement with those of Al-Rubeaan et $_{\text {al }}{ }^{(2)}$ and Hussein et al. $^{(16)}$ who found that YKL-40 has a significant positive correlation with $\mathrm{HbA1c}$. Moreover, there was a positive and none significant correlation between YKL-40 and albumin /creatinine ratio in the macroalbuminuric group.

Rathcke $\boldsymbol{e t}$ al. ${ }^{\left({ }^{9}\right)}$ found that there is a correlation of YKL-40 with urinary albumin to creatinine ratio (UACR) in the total group of participants. This correlation was not significant in any of the different subgroups.

The present work is a cross-sectional trial. Prospective trials are needed to confirm our results.

\section{CONCLUSION}

We conclude from our data that serum levels of YKL-40 were significantly higher in type 2 diabetic patients than in normal patients, and the levels of YKL-40 gradually were increased with increasing urinary albumin excretion rate. It was an independent factor associated with albuminuria in the early stage of nephropathy in type 2 diabetes and might have a useful role as a noninvasive marker for early diabetic nephropathy detection.

\section{REFERENCES}

1. American Diabetes Association (2014): Diagnosis and classification of diabetes mellitus. Diabetes Care, 37 (1): 81-90.

2. Al-Rubeaan K, Siddiqui K, Al-Ghonaim M et al. (2017): Assessment of the diagnostic value of different biomarkers in relation to various stages of diabetic nephropathy in type 2 diabetic patients. Scientific Reports, 7 (1): 1-9.

3. Rathcke C, Raymond I, Kistorp C et al. (2010): Lowgrade inflammation as measured by levels of YKL-40: association with an increased overall and cardiovascular mortality rate in an elderly population. International Journal of Cardiology, 143 (1): 35-42.
4. American Diabetes Association (2020): Classification and diagnosis of diabetes: standards of medical care in diabetes-2020. Diabetes Care, 43: 14-31.

5. Caraway W, Watts $\mathbf{N}$ (1986): Textbook of Clinical Chemistry, WN Saunders Co., Philadelphia, PA., Pp. 784-789.

6. Zhao X, Tang R, Gao B et al. (2007): Functional variants in the promoter region of chitinase 3-like 1 (CHI3L1) and susceptibility to schizophrenia. The American Journal of Human Genetics, 80 (1): 12-18.

7. Ober C, Tan Z, Sun Y et al. (2008): Effect of variation in CHI3L1 on serum YKL-40 level, risk of asthma, and lung function. New England Journal of Medicine, 358 (16): 1682-1691.

8. Knapp R, Miller C (1992): Clinical epidemiology and biostatistics. The national medical series for independent studies. Williams and Wilkins. MASS Publishing Company. Baltimore, USA., Pp: 98:521.

9. Rathcke C, Persson F, Tarnow L et al. (2009): YKL40, a marker of inflammation and endothelial dysfunction, is elevated in patients with type 1 diabetes and increases with levels of albuminuria. Diabetes Care, 32 (2): 323-328.

10. Perkins B, Ficociello L, Ostrander B et al. (2007): Microalbuminuria and the risk for early progressive renal function decline in type 1 diabetes. Journal of the American Society of Nephrology, 18 (4): 1353-1361.

11. Yokoyama H, Sone H, Oishi $M$ et al. (2008): Prevalence of albuminuria and renal insufficiency and associated clinical factors in type 2 diabetes: the Japan Diabetes Clinical Data Management study (JDDM15). Nephrology Dialysis Transplantation, 24 (4): 1212 1219.

12. Elsayed M, El Badawy A, Omar R et al. (2019): Serum cystatin $\mathrm{C}$ as an indicator for early detection of diabetic nephropathy in type 2 diabetes mellitus. Diabetes \& Metabolic Syndrome: Clinical Research \& Reviews, 13 (1): 374-381.

13. Umapathy D, Dornadula S, Krishnamoorthy E et al. (2018): YKL-40: A biomarker for early nephropathy in type 2 diabetic patients and their association with inflammatory cytokines. Immunobiology, 223 (11): 718-727.

14. Sueud T, Hadi N, Abdulameer R et al. (2019): A. Assessing urinary levels of IL-18, NGAL, and albumin creatinine ratio in patients with diabetic nephropathy. Diabetes \& Metabolic Syndrome. Clinical Research \& Reviews, 13 (1): 564-568.

15. Han J, Ma X, Yu L et al. (2015): Correlation between serum YKL-40 levels and albuminuria in type 2 diabetes. Genet Mol Res., 14 (4): 18596-603.

16. Hussein A, Rizk M, Ahmed A et al. (2016): Association of serum YKL-40 levels with urinary albumin in Egyptian patients with type 1 diabetes mellitus. Int J Adv Res Biol Sci., 3 (9): 1-12. 